\title{
LUCAS BURKART
}

\section{Verworfene Inspiration. \\ Die Bildgeschichte Percy Ernst Schramms und die Kulturwissenschaft Aby Warburgs}

\author{
1. Aby Warburg
}

"Warburgs Geltung - Mehr noch als frühere Jahrhunderte scheint das jetzt zu Ende gehende mit einer merkwürdigen Ehrfurcht auf die beiden Jahrzehnte seines Beginns zurückzublicken, als seien in den Umbrüchen der Jahrhundertwende unwiederbringliche Energien freigesetzt, die für ein ganzes Saeculum reichen sollten. Wie Max Weber für die Sozialwissenschaften, Sigmund Freud für die Psychoanalyse und Albert Einstein für die Physik, so ist auch Aby Warburg (1866-1929) für die Geschichte der Kunst und des Bildes eine beständige Herausforderung geblieben. Wenn das 20. Jahrhundert das des Bildes war, so gehört der Kunsthistoriker Warburg zu dessen herausragenden Forschern, weil ihm in der Intensität und der Skrupelhaftigkeit, mit denen er sich Kunstwerken, aber auch Alltagsbildern gewidmet hat, niemand gleichgekommen ist."1

Mit diesen Worten haben Horst Bredekamp und Michael Diers im Vorwort zur Studienausgabe der Gesammelten Schriften Warburgs den Hamburger Privatgelehrten als Begründer einer Kulturwissenschaft im Zeichen des Bildes und der Kunst gewürdigt; diese Einschätzung stieß am Ausgang des 20. Jahrhunderts auch jenseits von Kunst- und Bildwissenschaft auf breite Zustimmung. Die Theorie eines kulturellen Bildgedächtnisses, wie Warburg sie im Mnemosyne-Atlas entwickelt hatte, ist auch für die Geschichtswissenschaft, die in jüngerer Zeit 'die Erinnerung' und 'das Bild' gleichermaßen als Untersuchungs- und Analysegegenstand entdeckt hat, von hoher Aktualität. ${ }^{2}$ Bezeichnete sich Warburg selbst stets als 'Historiker' und verstand sein Anliegen, das 'Nachleben der An-

1 Warburg, Gesammelte Schriften, S. 5.

2 Le Goff Geschichte und Gedächtnis. Für die Geschichte des Mittelalters vgl. Geuenich/Oexle, Memoria in der Gesellschaft; Oexle, Memoria als Kultur. Die Literatur zu der als Iconic Turn bezeichneten Wende zum Bild ist kaum mehr zu übersehen. Für die Geschichtswissenschaft hierzu: Kaemmerling, Ikonographie und Ikonologie; Tolkemitt/Wohlfeil, Historische Bildkunde; Haskell, Die Geschichte und ihre Bilder; Oexle, Der Blick auf die Bilder. 
tike' in den europäischen Kulturen zu erkunden, von allgemeinem historischem Interesse, wurden seine Anregungen in der deutschsprachigen Geschichtswissenschaft nur sehr zögerlich aufgegriffen Erst mit der Renaissance der Kulturgeschichte seit den 1980er Jahren geriet Warburgs Ansatz vermehrt in den Fokus der Ge schichtswissenschaft. ${ }^{3}$

Die Diskrepanz zwischen Warburgs Selbstwahrnehmung als Historiker, der sich einer historischen Frage verschrieben hatte, und der erst mit großer Verzögerung erfolgten Rezeption durch die Fachhistorie bietet Anlass, nach den wissenschaftsgeschichtlichen Gründen und Voraussetzungen hierfür zu fragen. Diese Frage ließe sich in ganz unterschiedlicher Weise erörtern. Als Ideengeschichte, als Geschichte der wissenschaftlichen Institutionen, als personengeschichtliche Untersuchung oder als Geschichte politischer Umwälzungen, durch die die von Aby Warburg begründete Forschungsbibliothek im Dezember 1933 von Hamburg ins Londoner Exil gezwungen worden war, was die Rezeption Warburgs in der deutschsprachigen (Geschichts-)Wissenschaft natürlich nachhaltig beeinflusst hat. Ich möchte hier keine diese Möglichkeiten verfolgen, sondern Verknüpfung unterschiedlicher Aspekte vornehmen, die man als Kulturgeschichte der Wissenschaft bezeichnen könnte oder als 'Problemgeschichte', wie Otto Gerhard Oexle vorgeschlagen hat. Zum einen lassen sich somit über einen längeren Zeitraum hinweg sowohl Veränderungen, aber auch Kontinuitäten wissenschaftlicher Praxis beobachten, zum anderen werden nicht nur die in der Wissenschaft entwickelten Ideen, sondern auch die diesen zugrunde liegenden Fragestellungen sichtbar. ${ }^{4}$ So verbinden sich im Folgenden persönliche Prägungen, institutionelle Bedingungen des Wissenschaftsbetriebs, Kontinuitäten mediävistischer Historiografie und politische Zusammenhänge zu einer Deutung nicht nur der lange ausgebliebenen Rezeption Warburgs in der Geschichtswissenschaft, sondern deren Konsequenz für die Entwicklung einer methodisch geleiteten historischen Bildforschung.

\footnotetext{
Zur Rezeption Warburgs in der modernen Kulturgeschichte vgl. Roeck, Psychohistorie. Die frühe, bezeichnenderweise nicht deutsche Ausnahme bleibt: Ginzburg, Da Warburg a E. H. Gombrich. Zur Rezeption Warburgs in der französischen Geschichtswissenschaft vgl. Raulff, Parallel gelesen.
} Oexle, Geschichtswissenschaft im Zeichen; Oexle, "Staat" - "Kultur" - "Volk".

\section{Hamburger Inspirationen}

Die Veröffentlichung von Percy Ernst Schramms (1894-1970) Studie Das Herrscherbild in der Kunst des frühen Mittelalters in der Reihe "Vorträge der Bibliothek Warburg" von 1924 dokumentiert bis heute gut sichtbar eine Verbindung zwischen dem Mittelalterhistoriker Schramm und dem Kreis der Forscher um Aby Warburg und dessen Bibliothek. Die Publikation beruht auf einem Vortrag, den Schramm am 30. Dezember 1922 in den Räumen der Bibliothek Warburg gehalten hatte. Zur Vorbereitung des Vortrags war er von seinem Studienort Heidelberg für ein paar Monate in seine Heimatstadt zurïckgekehrt, wo er im Warburg-Kreis "etwas geistige Auffrischung durch Sie [F. Saxl, L.B.], die Bibliothek usw." suchte. ${ }^{5}$ Offenbar fand er diese auch, sodass er bereits im Oktober begeistert an Warburg, der sich in Kreuzlingen in die Behandlung von Ludwig Binswanger begeben hatte, schrieb:

"Ich geniesse jetzt wirklich Ihre Bibliothek, wo ich alles bei der Hand habe, und ich staune langsam immer mehr, [...] wofür Sie schon früher Interesse gehabt haben, als manche heute viel ventilierte Dinge noch gar nicht aktuell waren. Es lässt sich großartig mit dem Material arbeiten!"

Hinzu fügte er: "Zu nett ist natürlich, dass ich in Saxl und $\mathrm{Pa}-$ nofsky immer zwei mit mir befreundete Kunsthistoriker zur Hand habe." ${ }^{\prime 6}$

In der gedruckten Fassung bedankte sich Schramm entsprechend bei diesen Forschern sowie bei dem Heidelberger Archäologen Ludwig Curtius: "Herr Prof. Dr. A. Warburg möge aus diesem Aufsatz ersehen, dass dem Verfasser die Anregungen, die er von ihm empfangen hat, auch in seiner engeren Disziplin von Bedeutung geworden sind. Wärmsten Dank für Auskünfte, Ratschläge und Hinweise schuldet der Verfasser Prof. Dr. L. Curtius, Dr. E. Panofsky und besonders Dr. F. Saxl." ${ }^{17}$ Die Verbundenheit zwischen Schramm und der Bibliothek Warburg war damals gegenseitig. Saxl hatte das Vortragsprogramm bereits im Frühjahr 1922 abgesprochen und Schramm als Redner "zu einem unsere Bibliothek ja sehr interessierenden Thema" bezeichnet und einige Tage

\footnotetext{
WIA [Warburg Institute Archive], GC [General Correspondance] 1922, P. E. Schramm an F. Saxl, Heidelberg, 3. April 1922.

6 WIA, GC 1922, P. E. Schramm an A. Warburg, Hamburg, 5. Okt. 1922.

Schramm, Das Herrscherbild.
} 
nach dem Vortrag schrieb er nach Kreuzlingen: "Der Vortrag von Percy war glänzend besucht und ganz anständig. [...] Er [Schramm] ist wirklich herausgeboren aus den Räumen der Bibliothek Warburg."8

Schramms Verbundenheit zu Aby Warburg reichte vor den Ersten Weltkrieg zurück; ihre Wurzeln hat sie letztlich im gesellschaftlichen Umgang des hanseatischen Grossbürgertums. Schramm begegnete Warburg bereits als Gymnasiast in seinem Elternhaus, in dem dieser als gern gesehener Gast häufig zugegen war. Schramms Vater, Max, der 1912 zum Senator gewählt wurde und später zum Bürgermeister (1925-1928) aufstieg, war Aby Warburg aber nicht nur freund- und gesellschaftlich, sondern auch wissenschaftspolitisch verbunden. Gemeinsam arbeiteten sie auf die Gründung einer Hamburger Universität hin - ein Projekt, dessen zäher Verlauf Warburg manch sarkastischen Kommentar über die Hamburger Bürgerschaft entlockte. Konnte Max Schramm Warburgs Anliegen an dieser Stelle politisch befördern, gelangte er seinerseits über dessen Vermittlung ins Kuratorium des Kunsthistorischen Instituts in Florenz. 1926 schließlich erwog Warburg, den damaligen Bürgermeister ins Kuratorium seiner eigenen Bibliothek zu berufen: wie er sagt, "wegen seines Sinnes für das 'wo geistig was dran ist'. Hat doch den Sinn für die Hauptsache." ${ }^{9}$ Die befreundeten Familien Schramm und Warburg pflegten nicht nur gemeinsamen gesellschaftliche Umgang, sondern waren in den großbürgerlichen Hamburger Stadtteilen Harvestehude und Eppendorf auch Nachbarn, deren Stadtvillen nur wenige Gehminuten voneinander entfernt lagen. ${ }^{10}$

Ausgehend von dieser für das großbürgerliche Hamburg bezeichnenden Konstellation entstand zwischen Warburg und dem jungen Schramm eine weit über gesellschaftliche und politische Gemeinsamkeiten hinausreichende, freundschaftliche Lehrer-Schüler Beziehung, wie sie der Privatgelehrte etwa auch mit Carl Georg 8 WIA, GC 1923, Mappe "Saxl/Warburg", F. Saxl an A. Warburg, Hamburg, 4.
Jan. 1923.

9 Warburg, Tagebuch, S. 26.

10 Das Wohnhaus und die daran angebaute Kulturwissenschaftliche Bibliothek Warburgs liegen in der Heilwigstrasse 114 und 116, während das Elternhaus Percy Ernst Schramms am Frauenthal 29 lag. In seiner Familiengeschichte hat Schramm das Haus und die damit verbundene, luxuriöse Lebensweise beschrieben. Vgl. Schramm, Neun Generationen, S. 420-426.
Heise pflegte." Warburg förderte Schramms historische Interessen, die sich zunächst auf die Geschichte hanseatischer Familien konzentrierte, indem er ihn in seiner Privatbibliothek arbeiten ließ; zugleich verteidigte er die historischen Interessen des Bürgersohns gegenüber den beunruhigten Eltern. Auch als Schramm 1914 in Freiburg das Studium der Geschichte aufnahm, hielt der Kontakt zu seinem Mentor an. Dieser hatte ihn mit Empfehlungsschreiben an befreundete Freiburger Kollegen, etwa an den Kunsthistoriker Wilhelm Vöge, den Religionswissenschafter Richard Reitzenstein oder den Historiker Heinrich Finke ausgestattet. Die Distanz zwischen Freiburg und Hamburg überwanden Mentor und Schüler mit regelmäßigen und manchmal ausführlichen Schreiben. Auch während des Ersten Weltkrieges, für den sich Schramm in den ersten Tagen als Freiwilliger gemeldet hatte, brach die Korrespondenz nicht ab.

Als Warburg nach dem Kriegsende einen psychischen Zusammenbruch erlitt, begab er sich zunächst in eine Privatklinik, wo Schramm ihn nach seiner Rückkehr nach Hamburg im Dezember 1918 ebenso wie Heise regelmäßig besuchte und sich mit ihm über wissenschaftliche Themen unterhielt, wenn auch nur, um dem verehrten Lehrer dessen Wahnvorstellungen vorübergehend erträglicher zu machen. Die Gesellschaft war Warburg ganz offensichtlich angenehm und so wünschte er 1920, dass ihn auf seiner Reise von Hamburg nach Jena, wo er sich in ein Sanatorium begeben wollte, neben seinem Arzt und seiner Frau Mary Percy Ernst Schramm begleiten sollte; und schließlich erhoffte sich Warburg auch für die Reise von Jena in die Kreuzlinger Klinik des Psychoanalytikers Ludwig Binswanger die Begleitung Percy Ernst Schramms. Die Wertschätzung Warburgs beschränkte sich jedoch nicht nur auf die zwischenmenschliche Beziehung. Er schätzte Schramm als zuverlässige und starke Persönlichkeit ein; entsprechend hatte er ihn in einem vor seiner Abreise nach Jena aufgesetzten Testament für den Fall seines Todes als Mitglied des Bibliothekskuratoriums vorge-

11 Diese Beziehung lässt sich aus der recht umfangreichen Korrespondenz zwischen den beiden ablesen, die sich heute im Warburg Archiv in London bzw. im schrammschen Familiennachlass in Hamburg befindet. Vgl. Thimme, Percy Ernst Schramm, S. 44-61. Die Briefe Schramms an Warburg wurden später sowohl von diesem für ein Publikationsvorhaben als auch von Saxl für die von ihm geplante Warburg-Biografie erneut gelesen. In der "intellektuellen Biographie" Warburgs von Gombrich, welche die Vorarbeiten Saxls verwendete, erscheint der Name Schramm hingegen nicht ein einziges Mal. 
sehen. Ganz offensichtlich traute er dem damals mit Abstand jüngsten und in diesem Gremium einzigen nicht Promovierten zu, einen Beitrag zum Fortbestand seines Lebenswerks leisten zu können. ${ }^{12}$

Während Warburgs Abwesenheit wurde die Leitung der Bibliothek und der wissenschaftlichen Aktivitäten Fritz Saxl übertragen. In der Person von Max Warburg verlangte die Familie, die sich von Aby immer wieder davon überzeugen ließ, die enormen Kosten zu tragen, die Bibliothek und Forschung verursachten, nun ihrerseits, vom Leiter ad interim schriftlich über die wissenschaftlichen Ziele und deren Folgekosten unterrichtet zu werden. Saxl kam der Aufforderung nach und erstellte eine "Denkschrift" sowie einen Haushaltsplan. In einem Rückblick auf diese Herausforderung erwähnt Saxl ausdrücklich die tatkräftige Mithilfe Schramms, die dazu beigetragen hatte, "dass ich das große Budget gleich bekam." ${ }^{13}$ Wie Warburg es sich vorgestellt hatte, trug Schramm dazu bei, das in der Bibliothek betriebene Projekt des "Nachlebens der Antike" mitzutragen und fortzuführen. Eine Bemerkung Saxls lässt vermuten, dass Schramm hierbei vor allem seine Kenntnisse hanseatischgroßbürgerlicher Kultur sowie entsprechende, adressatengerechte Formulierungen beisteuerte und weniger für die inhaltliche Ausgestaltung des Forschungsvorhabens zuständig war; dennoch ist unbestritten, dass Schramm aufs Nächste mit Ziel und Methode der Bibliothek Warburg vertraut war und sich ihr in seiner eigenen Forschung teilweise auch verschrieb. ${ }^{14}$

Diese Vertrautheit gründete mehr als auf allem anderen auf der langjährigen Beziehung zwischen Schramm und Warburg, den Gesprächen, die sie miteinander führten und den Ratschlägen, die jener von diesem erhielt. Die Begegnung mit Warburg hatte somit auf Schramm großen Einfluss und prägte sein wissenschaftliches Profil. Ihr verdankt sich Schramms interdisziplinäre Sichtweise auf Geschichte, besonders sein Interesse an Bildern, sowie eine damals in der Geschichtswissenschaft unübliche Herangehensweise an Bilder. Drei Aspekte, die Warburgs Einfluss unübersehbar werden

12 Thimme, Percy Ernst Schramm, S. 98 f.

13 WIA, GC 1921, F. Saxl an W. Printz, Hamburg, 12. Dez, 1921. Zur Situation der Bibliothek Warburg vor ihrer Emigration 1933 nach London und dem Zusammenhang von Wissenschaft und finanzieller Ausstattung vgl. Burkart, "Die Träumereien".

14 Thimme, Percy Ernst Schramm, S. 102 f. lassen, waren dabei für Schramms frühe Forschung von besonderer Bedeutung. Erstens lokalisierte er Bilder nicht mehr primär im Bereich entweder der Kunstgeschichte oder der historischen Hilfswissenschaften; vielmehr erachtete er sie als genuinen Teil historischer Forschung und verstand sie als Quellen eigenen Rechts, die Schriftquellen nicht nur illustrierten, sondern "diese, wo sie fehlen, sogar ersetzen können."15 Damit überschritt er zweitens Gattungsgrenzen, die durch die Hilfswissenschaften etabliert worden waren. Diplomatik, Heraldik, Sphragistik und Numismatik unterschieden ihre Gegenstände scharf und stellten deren Analyse in den Dienst der Geschichte, eröffneten jedoch kaum einen gemeinsamen Blick auf einen historischen Bilderkorpus. $\mathrm{Zu}$ diesem bereits umfangreichen Korpus, den Schramm nun umfassend in den Blick nahm, schlug er den Bildbestand der "hohen Kunst" hinzu, der bis anhin meist als Zuständigkeitsbereich der Kunstgeschichte erachtet worden war. Drittens schließlich überschritt er mit der Frage nach dem Wandel eines Bildtypus wie des Herrscherbildes die Epochengrenze und blickte in diachroner Perspektive auf die Geschichte. In den einführenden Bemerkungen zu seiner Studie zum Herrscherbild im Frühmittelalter von 1924 fordert Schramm all dies programmatisch. Die Anleihen bei dem bekannten Aufruf, mit dem Warburg 1912 seinen Vortrag zum Freskenzyklus im Palazzo Schifanoia ausklingen ließ, sind kaum zu übersehen:

"Kommilitonen! [...] Ich hoffe durch die Methode meines Erklärungsversuches der Fresken im Palazzo Schifanoja zu Ferrara gezeigt zu haben, dass eine ikonologische Analyse, die sich durch zeigt zu haben, dass eine ikonologische Analyse, davon abschrecken lässt, Antike, Mittelalter und Neuzeit als zusammenhängende Epoche anzusehen, noch davon, die Werke freister und angewandtester Kunst als gleichberechtigte Dokumente des Ausdrucks zu befragen, dass die Methode, indem sie sorgfältig sich um die Aufhellung einer einzelnen Dunkelheit bemüht, die großen allgemeinen Entwicklungsvorgänge in ihrem Zusammenhange beleuchtet." ${ }^{16}$

Jenseits methodischer Anregungen ließ sich Schramm auch vom Arbeitsstil inspirieren, wie er in der Kulturwissenschaftlichen Bibliothek an der Heilwigstrasse gepflegt wurde. ${ }^{17}$ Die Bildanordnun-

15 Schramm, Das Herrscherbild, S. 145.

16 Warburg, Italienische Kunst, S. 478 f.

17 Dabei muss der Arbeitsstil in gewisser Weise als Ausdruck der warburgschen Methode gelten, lässt sich also von dieser letztlich nicht trennen. 
gen des Mnemosyne-Atlas waren für Warburg weniger abschlieBendes Forschungsergebnis als ein Arbeitsinstrument, mit dem die Erörterung der ihn beschäftigenden Frage nach dem 'Nachleben der Antike' immer aufs Neue möglich wurde. Der Bilderatlas war im Grunde das visuelle Pendant zur Bibliothek, die Saxl 1930 "als Problemsammlung für die Erforschung des Nachlebens der Antike" charakterisierte. ${ }^{18}$ Schramms erste Buchpublikation, Die deutschen Kaiser und Könige in Bildnissen ihrer Zeit von 1928 war diesem Stil verpflichtet. Den Sommer 1927 verbrachte Schramm in Hamburg, wo er die Arbeit nochmals intensiv mit Saxl besprach, bevor er sein imprimatur gab. ${ }^{19}$ Die Untersuchung umriss in der gebotenen Sammlung und Ordnung des Materials eher eine Fragestellung; sie ist weniger Synthese und Interpretation als annotierter Bilderkatalog früh- und hochmittelalterlicher Herrscherporträts in unterschiedlichen Bildmedien.

Dieser Vorgehensweise verschrieb sich Schramm auch in weiteren Forschungsprojekten; seine Denkmale der deutschen Könige und Kaiser, in zwei Bänden 1962 und postum 1978 erschienen, und seine Herrschaftszeichen und Staatssymbolik (1954-1956) stehen in dieser Tradition. Dieses Vorhaben, das bis heute als Standardwerk gilt, hatte Schramm bereits lange vor seiner Fertigstellung als corpus regalitatis medii aevi, also als eine Materialsammlung zur Behandlung der Frage mittelalterlicher Herrschaft bezeichnet. Im Vorwort betont er, dass es in der Studie "darauf ankommt, einen allgemeinen Überblick zu gewinnen, Methoden zu erproben, Fragen zur Erörterung zu stellen und dadurch andere Forscher zur Stellungnahme und weiteren Nachsuche zu veranlassen". ${ }^{20}$

18 Saxl, Die Kulturwissenschaftliche Bibliothek, S. 332.

19 Warburg, Tagebuch, S. 132.

20 Schramm, Herrschaftszeichen und Staatssymbolik, S. VII. Für die Erarbeitung von raisonnierten Sammlungen bildlicher Quellenbestände stellt in der Zwischenkriegszeit der Kontakt zum Kreis der Bibliothek Warburg nicht den einzigen Anknüpfungspunkt dar. Im Umfeld der internationalen Historikerkongresse, aber letztlich von marginaler Bedeutung, kam es in der Zwischenkriegszeit immer wieder zu Initiativen, Bildquellen für die Geschichtswissenschaft fruchtbar zu machen. Die Bildung nationaler und internationaler ikonografischer Komitees wurde immer wieder angeregt, und Schrationaler ikonografischer fung nach Göttingen 1929 gemeinsam mit Karl Brandi auch involviert. Bereits 1928 waren Schramm und Brandi gemeinsam am Historikertag in Oslo, wo eine Kommission für Ikonografie gegründet wurde, und 1930 begründeten die beiden einen Deutschen Ikonographischen Ausschuss. Da in diesen Zirkeln in
Schließlich prägte auch Warburgs Ringen um eine angemessene Sprache den jungen Schramm. Auch wenn er es niemals zur Kunstfertigkeit warburgscher Wortschöpfung brachte, die im Ringen mit dem 'Problem' eine schöpferische Kraft gewann, quälte ihn wie seinen Lehrer ein Unbehagen der Wissenschaftssprache gegenüber. Gerade wenn es um das Verhältnis von Text und Bild ging, stand nach Schramms Ansicht keine adäquate Begrifflichkeit bereit. Es fehlten die notwendigen 'Greifwerkzeuge', wie Warburg formulierte. Dass Warburg nicht nur bei diesem Unbehagen, sondern auch bei neu geschaffenen Termini Pate stand, ist an Begriffen wie Tendenzbild ${ }^{21}$ oder Devotions- und Majestätsforme ${ }^{22}$ zu erkennen, mit denen Schramm christliche bzw. antike Schemata bezeichnete, welche die bildende Kunst für mittelalterliche Herrschaftszeichen tradierte.

Es zeigt sich hier erneut das Erbe Warburgs, der zwischen der Sprache und dem Gegenstand der Wissenschaft keine Trennung vornehmen wollte. So verwies die Formel auf eine auf Warburg und Schramm gleichermaßen zutreffende Blickweise, die Bilder als Schemata verstand und den Fokus auf deren formalen Ursprünge und Wandel richtete. Dieses Verständnis prägte nicht zuletzt auch Schramms Verhältnis zur Kunstgeschichte, die er ja auf Anraten Warburgs bereits in Freiburg bei Wilhelm Vöge, dann aber vor allem in München bei Heinrich Wölfflin studierte. In der Einleitung zu seinen Herrscherbildnissen grenzt er sich explizit von der damals aktuellen, kunsthistorischen Frage nach der Ähnlichkeit zwischen Herrscher und Herrscherbild ab, entwickelte ikonografische Typologien von Herrscherbildern und eröffnete damit neue Perspektiven. Dies hatte jedoch auch seinen Preis; die Berücksichtigung der Ästhetik von Bildern etwa war mit diesem Ansatz kaum zu vereinbaren. In seiner Einleitung zu Herrschaftszeichen und Staatssymbolik schrieb Schramm dort von für sein Er-

Fragen der Terminologie und Methode ikonografischer und ikonologischer Analyse jedoch nie Einigkeit erzielt wurde, war jenseits unterschiedlicher Formen der Materialsammlung und -ordnung kaum etwas zu erreichen; das Fehlen einer methodengeleiteten Fragestellung bewirkte nicht zuletzt, dass diese Sammlungen sich innerhalb der Gattungsgrenzen bewegten und dem Porträt bzw. der Porträtmalerei entsprechend ein besonders hoher Status beigemessen wurde. Die Ausstrahlung dieser Initiativen auf das Fach blieb somit sehr gering. Vgl. hierzu den Beitrag von Jäger in diesem Band.

21 Schramm, Das Herrscherbild, S. 145.

22 Schramm, Herrschaftszeichen und Staatssymbolik, S. 16. 
kenntnisinteresse "völlig ungenügenden Darstellungen, wo offensichtlich ein rein ästhetischer Antrieb wirksam war. [...] Ästhetik", so Schramm weiter, "kann sich dort entfalten, wo es nicht um Wirklichkeit geht". ${ }^{23}$ Auch hierin zeigt sich Warburgs Einfluss, der für seine Frage nach dem antiken Nachleben Kontinuität und Wandel bildlicher Formen ebenfalls nicht unter dem Blickpunkt einer Ästhetik untersuchte.

Zusammenfassend lässt sich feststellen, dass Aby Warburg und die Kulturwissenschaftliche Bibliothek Warburg den jungen Percy Ernst Schramm nachhaltig beeinflusst und geprägt hatten; durch sie wurde ihm das Bild als Gegenstand historischer Forschung nahegebracht, ein Sensorium für Sprache und Begrifflichkeit erweckt und Arbeitsweisen und -techniken eröffnet, die damals außerhalb dieses Kreises wenig verbreitet waren; all dies war eng mit einem methodischen Verständnis verbunden. Was Schramm vom Kreis der Bibliothek Warburg als Anregung aufnahm und lernte, bot gute Voraussetzungen dafür, dass sich die Geschichtswissenschaft konstruktiv mit Bildquellen beschäftigen konnte. Die Arbeiten und das methodische Verständnis des jungen Percy Ernst Schramm waren hierfür ein Versprechen, ein Versprechen allerdings, das auch Schramm selbst nicht zu halten vermochte.

\section{Eine Geschichte des Mittelalters und die akademische Sozialisation}

Hatte Aby Warburg die historischen Interessen des Gymnasiasten und Studenten Schramm stets gefördert und gegenüber dem besorgten Vater verteidigt, wuchs Schramms Entscheid, sich der Geschichte des Mittelalters zuzuwenden, unabhängig von Warburg. Dessen Interesse an der Kunst und Geschichte des Mittelalters war stets beschränkt und mittelbarer Art. Warburg sah im Mittelalter letztlich eine Etappe auf der 'Wanderstrasse' eines antiken Bildkataloges, der erst in der Frührenaissance wieder entdeckt, d. h. sowohl seiner Form als auch seinem Inhalt nach wieder der antiken Bedeutung gemäß verstanden wurde - dieser Frage galt sein Interesse. $^{24}$ Auch Fritz Saxl, dessen zweibändiges Werzeichnis illust-

\footnotetext{
23 Schramm, Herrschaftszeichen und Staatssymbolik.

${ }^{24}$ Gombrich, Aby Warburg, S. $101 \mathrm{f}$.
}

rierter Handschriften zur Astrologie und Mythologie, 1915 und 1927 bei Winter in Heidelberg erschienen, bis heute einen wichtigen Beitrag zur Mittelalterforschung darstellt, sah im Mittelalter einen Abschnitt der langen Geschichte des 'antiken Nachlebens'. Sein Interesse galt weniger der Epoche per se als den Bedeutungsverschiebungen, die sie hinsichtlich der Tradierung eines antiken Bildrepertoires hervorbrachte. Im Kreis der Bibliothek Warburg wies die mittelalterliche Geschichte stets einen doppelten Fluchtpunkt auf - die Antike und ihre Wiederentdeckung im $\mathrm{Hu}$ manismus und in der Renaissance -, doch selbst geriet sie nie ins Zentrum des Interesses.

Schramms Interesse an der Geschichte des Mittelalters musste also anders angeregt worden sein. Eigener Aussage zufolge hatte ihn die Lektüre von Karl Hampes Deutsche[r] Kaisergeschichte in der Zeit der Salier und Staufer, 1909 erstmals in Leipzig erschienen, tief beeindruckt. Schramm fühlte sich wie viel seiner Generation damals von einer Darstellung des hochmittelalterlichen Reiches als glorreicher Vergangenheit deutscher Größe, wie sie Hampe in seinem Buch bot, angesprochen. Dokumente aus seinem Nachlass zeigen, dass der junge Schramm auch gegenüber neo-romantischer Mittelalter-Schwärmerei sehr empfänglich war. Auf Reisen, die Schramm gemeinsam mit Freunden zu den historischen Kulturstätten mittelalterlicher Geschichte unternahm, wurde diese Sicht als 'direkte Begegnung' mit der Geschichte verstärkt. Doch das Gefühl, im mittelalterlichen Reich eine bedeutungsvolle deutsche Vergangenheit zu entdecken, kontrastierte scharf mit der Erfahrung der Kriegsniederlage oder war gar von dieser zusätzlich angeregt. Noch in den 1960er Jahren hielt Schramm an diesem Zusammenhang fest und formulierte: "In meinem Bewusstsein kapitulierte das 20. Jahrhundert vor der Kaiserherrlichkeit des Mittelalters." ${ }^{25}$ Die Geschichte des Mittelalters schien ihm Sicherheiten zu bieten, die ihm als Angehörigem der Frontgeneration abhanden gekommen waren. "Als ich nach dem Ersten Weltkrieg in abgeänderter Uniform meine Studien wieder aufnahm, kam folgendes hinzu: blickte man auf unsere jüngste Vergangenheit zurück, war schlechthin alles infrage gestellt; das Mittelalter war jedoch vom Schwanken der Urteile nur mittelbar berührt, bot also einen festen Boden. Dabei schwang der Gedanke mit, dass die Rückbesinnung

\footnotetext{
25 Thimme, Percy Ernst Schramm, S. 52.
} 
auf die frühere Vergangenheit uns vielleicht für die Zukunft Halt zu bieten vermöge." ${ }^{26}$ Zugleich schien die Größe der 'deutschen Geschichte des Mittelalters' angesichts der Monumentalität der eigenen Kriegserfahrung einen adäquaten Studiengegenstand zu bieten. ${ }^{27}$

In München, wohin Schramm nach Kriegsende zum Studium gewechselt war, richtete er seine Aufmerksamkeit zunächst auf die Frage nach dem "Wesen des Staates", dann auf diejenigen nach dem "Wesen des mittelalterlichen Staates". Dies führte, auch angeregt durch Wölfflins 'Wesensschau', dessen Vorlesung Schramm besuchte, unweigerlich zu der Frage nach dem 'deutschen Wesen', dem 'deutschen Geist' und dem Wert der deutschen Kultur, zu dem sich damals zahlreiche Intellektuelle äußerten. ${ }^{28}$ Von München wechselte Schramm 1921 nach Heidelberg, wo Karl Hampe lehrte, bei dem er nur ein Jahr später das Doktorexamen ablegte. In diese Zeit fielen auch die Begegnung mit Harry Bresslau, den Schramm bei dessen Editionsprojekten für die MGH unterstützte, und mit Friedrich Baethgen, ebenfalls Monumentist, bevor 1924 die Berufung zum Extraordinarius in Heidelberg erfolgte. In München und Hamburg bewegte sich Schramm somit in einem ganz anderen Ambiente als in Hamburg; im Gegensatz zur Hansestadt, in der es ja erst 1919 überhaupt zur Gründung einer Universität kam, waren München und Heidelberg beides spätmittelalterliche Universitätsgründungen. Aber nicht nur die Institution selbst, sondern auch das Fach der mittelalterlichen Geschichte blickte hier auf eine alte Tradition, die bis in die Gründungsphase der akademischen Mediävistik zu Beginn des 19. Jahrhunderts zurückreichte und in den Arbeitsstellen der MGH in Heidelberg und München ihr Fortleben hatten. Schramm begegnete hier also einer Forschungstradition, die ihre erste Aufgabe in der kritischen Editionen von Quellentexten sah und sich in zweiter Linie einem politik- und verfassungsgeschichtlichen Ansatz verpflichtet fühlte; das Augenmerk war somit meist 'auf die Darstellung der staatlichen Entwicklung' gerichtet. ${ }^{29}$

26 Schramm, Kaiser, Könige und Päpste, S. 7.

$27 \mathrm{Zu}$ persönlichen Äußerungen Schramms hierzu vgl. Thimme, Percy Ernst Schramm, S. $90 \mathrm{f}$.

28 Oexle, Geschichtswissenschaft im Zeichen des Historismus, S. 177.

29 Hampe, Deutsche Kaisergeschichte. S. V. Schramm sollte sich noch lange Zeit mit dieser Frage auseinandersetzen. Vgl. die Einleitung in: Schramm, Herrschaftszeichen und Staatssymbolik, S. I.
Diese Forschungstradition prägte aber nicht nur die Sicht der Fachhistorie, sondern war weit darüber hinaus wirksam. Im Publikationserfolg der Kaisergeschichte von Schramms Doktorvater, Karl Hampe, spiegelt sich die Vorstellung eines 'deutschen Mittelalters' und ihre anhaltende Gültigkeit bis in die zweite Nachkriegszeit - möglicherweise auch darüber hinaus. Erstmals 1909 in Leipzig erschienen, erfuhr die Darstellung bis 1929 sechs Auflagen. Nach Hampes Tod 1936 unterzog sie Friedrich Baethgen, der 1913 bei Hampe über die Regentschaft Innozenz III. im Königreich Sizilien promoviert hatte, einer Überarbeitung und brachte sie bis 1969 in sechs weiteren Auflagen zum Druck, deren letzte bis 1985 in fünf Nachdrucken wieder aufgelegt wurde. ${ }^{30}$

Der Frage nach Genese und Bedeutung des Staates begegnete Schramm während seines Studiums in München und Heidelberg; gestellt wurde sie damals auch von der Mediävistik in ihrer Bedeutung auf den 'deutschen Sonderweg' hin; auch das war Teil der Forschungstradition und Historiografie, der Schramm begegnete und die ihn zeitlebens prägen sollte. In seinen gesammelten Aufsätzen, die 1968 bis 1971 unter dem Titel Kaiser, Könige und Päpste erschienen, zeigt sich die anhaltende Gültigkeit dieses Interesses und seiner Tradition deutlich. ${ }^{31}$ Das Interesse am Mittelalter als einer Vorgeschichte, aus deren Studium die eigentliche und zukünftige Bedeutung eines deutschen Staates zu ersehen und zu prognostizieren war, teilte Schramm mit vielen Historikern seiner Zeit. Das Mittelalter bot hier eine Fläche, auf die sich imperiale Macht und kulturelle Größe gleichermaßen projizieren ließen. ${ }^{32}$

30 Das gilt analog auch für Hampes Herrschergestalten des deutschen Mittelalters mit sieben Auflagen zwischen 1927 und 1955 sowie für sein Das Hochmittelalter mit vier Ausgaben zwischen 1932 und 1953, darunter einer "Frontausgabe" 1944. Vgl. Nagel, Im Schatten des Dritten Reichs, S. 51.

31 Schramm, Kaiser, Könige und Päpste.

32 Zur deutschsprachigen Mediävistik des ausgehenden 19. Jahrhunderts, ihrer Traditionen und Wirkungen vgl. Schieffer, Weltgeltung und nationale Verführung. Zu der Zwischenkriegszeit jüngst: Nagel, Im Schatten des Dritten Reichs, S. $39 \mathrm{f}$. Dabei zeigt die Autorin überzeugend, dass die historische Mittelalterforschung der Zwischenkriegszeit ihre Fragen, Themen und Methoden nicht nur aus dem 19. Jahrhundert und dem Trauma der Kriegsniederlage bezog, sondern sie als personelle, institutionelle, historiografische sowie methodische Kontinuitäten bis weit in die zweite Nachkriegszeit fortschrieb. Es geht ihr dabei nicht primär um die persönlichen Verstrickungen einzelner Historiker mit dem NS-Regime, sondern um die Strukturen der wissenschaftlichen Disziplin. Allgemein zur Tradierung und Adaption von Mittelalterbildern vgl. Groebner, Das Mittelalter hört nicht auf. 
Als Mediävist stand Schramm somit in ganz anderen Traditionen als die ihm befreundeten Forscher der Bibliothek Warburg. Hier eine aufs Universelle zielende Kulturgeschichte, dort das Interesse für den deutschen Sonderweg. Auch an der Bedeutung der Epoche selbst schieden sich die Geister. Schramm erkannte im Mittelalter nicht nur eine Zeit, in der "die Antike überwintert, und die wie ein Mensch geschlafen habe", wie er es noch im Nachwort seiner 1958 erschienenen Untersuchung Sphaira - Globus - Reichsapfel formulierte. Es lässt sich auch als Spitze gegen die 'Mittelalter-Verachtung' der Warburg-Forschung verstehen, wenn er dieser Studie den Untertitel hinzufügte: Ein Beitrag zum 'Nachleben der Anti$\mathrm{ke}^{\prime}{ }^{33}$ Wiederholt verwies er bereits in den 1920er und 1930er Jahren auf das mangelnde Interesse der Bibliothek Warburg am 'Nicht-Klassischen', d. h. an der 'nicht-klassischen' Epoche des Mittelalters und am 'Germanischen' - besonders hinsichtlich ihrer Bedeutung für die mittelalterliche Königs- und Kaiserherrschaft.

Schramms Interesse für die Geschichte Mittelalters erfolgte nicht nur jenseits seiner Kontakte zu Warburg, sondern seine Sicht auf das Mittelalter entsprach auch methodisch nicht dem Forschungsvorhaben seines ersten Mentors und Lehrers. Schramms Begegnung mit der mittelalterlichen Geschichte war geprägt von einer damals erfolgreich wirkenden Reichsmediävistik, die weit über die Grenzen universitärer oder akademischer Kreise ausstrahlte. Nach Schramms retrospektiv formulierter Aussage waren es über einen Zeitraum von 15 Jahren drei Momente, die in ihm das Interesse am Mittelalter weckten. Die Lektüre von Hampes Deutscher Kaisergeschichte, das Versprechen, "im Mittelalter Sicherheiten zu finden, die ihm als Angehörigem der Kriegsgeneration abhanden gekommen waren" und schließlich die Begegnung mit der "Darstellung des zwischen geistlichen und weltlichen Grossen thronenden Ottos III.", die ihn "ergriff" - eine Bezeichnung, die er in Anführungszeichen setzt, damit sie ihre Würde zurückerhält. ${ }^{34}$ Schramms Hinwendung zum Mittelalter war somit auch eine rückwärts gerichtete Flucht in eine Vergangenheit, deren Studium nach national-konservativer Vorgabe erfolgte und die dazu beitragen sollte, das Trauma des ersten Weltkriegs zu verarbeiten und die Frage nach

\footnotetext{
${ }_{34}$ Schramm, Sphaira - Globus - Reichsapfel, S. 707

34 Schramm, Kaiser, Könige und Päpste, Bd. 1, S. 8.
}

der kulturellen und politischen Bedeutung Deutschlands historisch $\mathrm{zu}$ beantworten und emphatisch zu überhöhen. ${ }^{35}$

Dieses Vorhaben fiel in der Bibliothek Warburg auf keinen fruchtbaren Boden, ja es lief deren Forschungsinteressen sogar entgegen. Versuchte Schramm bis Ende der 1920er Jahre seine beiden wissenschaftlichen Sozialisationen miteinander in Einklang zu bringen, die Frage nach dem Verständnis mittelalterlicher (Staats-)Herrschaft und ihrer symbolischen Repräsentation auch methodisch miteinander zu verbinden, entfernte er sich zusehends nicht nur vom Kreis der Bibliothek Warburg, sondern auch von dessen Methodenverständnis. ${ }^{36}$ Das Hauptanliegen Warburgs, die Frage nach dem 'Nachleben der Antike' hat der Mediävist Schramm nie zu seiner Leitfrage erhoben; vielleicht konnte und wollte er es nicht, weil zu ihrer Beantwortung das Mittelalter nicht ausreichend herangezogen wurde oder werden musste. Die Anregungen hingegen, die Schramm von Warburg und dessen Kreis an der Bibliothek erhielt, vermochte er auch als Mediävist konstruktiv für sich zu nutzen; indem er ganz nach Warburgs Verständnis das 'disziplinäre Grenzwächtertum' missachtete, bezog er nach der methodischen Vorgabe eines kulturellen Bildgedächtnisses Bild und Geschichte aufeinander. Doch konsequent folgte Schramm dem Verständnis Warburgs nur für eine kurze Zeit, bevor er sich zugunsten einer Mittelalterhistoriograhie, der dieses Methodenverständnis ebenso fremd war wie sie sich davon keine neuen Anregungen versprach, von ihm entfernte.

\section{Vom Symbol zum Zeichen}

Seiner bis heute grundlegenden Arbeit zu mittelalterlichen Herrschaftszeichen hat Schramm Überlegungen zum Gegenstand, zur

35. Hierzu gehört es auch, dass sich Schramm in seiner Heidelberger Zeit vom "erhabenen Ton" des George-Kreises angesprochen fühlte; hierbei spielten die zum George-Kreis zählenden, der gleichen Generation entstammenden Fachkollegen Wolfram von den Steinen (1892-1967) und Ernst H. Kantorowicz (1895-1963) eine entscheidende Rolle.

36 Ein Eintrag, den Warburg am 29. Mai 1928 in das Bibliotheks-Tagebuch schrieb, könnte als ein erster Hinweis auf diese Entfremdung zwischen Schramm und der Warburg-Schule verstanden werden: "Percy Schramm zeigt mir Durchschrift seines Buches der Romgedanke im Kaisertum und dessen Renovatio (bis zum Investiturstreit) oder so. Sichere ihm Annahme in den Studien zu; er schien Zweifel zu haben." Warburg, Tagebuch, S. 262. 
Methode und Terminologie des Vorhabens vorangestellt. "In diesem Buch", so die Vorrede, "ist der Ausdruck 'Insignie' vermieden, erst recht das wegen seiner Zweideutigkeit missliche Wort 'Symbol'". 1954 bis 1956 in drei Bänden erschienen, erscheint diese Skepsis gegenüber dem Symbolbegriff als vorsichtige Distanz gegenüber ideologischen Überschreibungen und Missbräuchen wissenschaftlicher Begriffe. Eine etwas ausführlichere Begründung für diesen Entscheid, mit der Schramm den dritten Band beschließt, revidiert diesen Eindruck jedoch. Der Grund, auf den Symbolbegriff $\mathrm{zu}$ verzichten, war ein doppelter. Erstens sah Schramm "den geistigen Boden, dessen Symbole bedürfen, in einer immer nüchterner werdenden Welt zusehends dürrer und unfruchtbarer". Zweitens "hat [der Begriff Symbol, L.B.] mittlerweile das Schicksal so vieler einst inhaltsgeladener Wörter geteilt, dass er durch die Verwendung als Umgangswort zu einer Hülse geworden ist, in die jeder stopft, was er meint. ${ }^{137}$ Hierin spiegeln sich zwei voneinander zu trennende Aspekte. Zum einen handelt es sich nicht um eine Problematisierung von Wissenschaftssprache angesichts der jüngsten deutschen Vergangenheit, sondern um eine rückwärts gewandte, der Moderne gegenüber kritische Äußerung; das zeigt sich deutlich an dem Motto, das Schramm dem ersten Band voranstellte und hier nochmals aufgriff: Goethes Versuch, das Wesen des Symbols zu fassen..$^{38}$ Zum zweiten reicht Schramms Ablehnung des Symbolbegriffs in die 1930er Jahre zurück und gründet in seiner Auseinandersetzung mit der Forschung Warburgs und der Kulturwissenschaftlichen Bibliothek.

Noch während Schramm um 1930 seine ordines-Studien betrieb, begann er sich mit den bei den Herrschererhebungen benutzten Objekten, den Insignien und Herrschaftszeichen zu beschäftigen; in seiner Untersuchung Die Krönung in Deutschland bis zum Beginn des Salischen Hauses (1028), erschienen 1935 in der Zeitschrift der Savigny-Stiftung für Rechtsgeschichte, verwies er auf die Bedeutung der Herrschaftssymbole und auf seine Absicht einer umfassenden Darstellung derselben. Dieses Projekt gehörte noch eindeutig in den Kontext, den Schramm mit der Bib-

37 Schramm, Herrschaftszeichen und Staatssymbolik, S. 1076.

38 "Es [das Symbol] ist die Sache, ohne die Sache zu sein, und doch die Sache, ein im geistigen Spiegel zusammengezogenes Bild und doch mit dem Gegenstand identisch." Goethe, Nachträgliches zu Philostrats Gemählden, S. 141 (vgl. hierzu auch Knauer in diesem Band). liothek Warburg, allen voran mit Saxl verband. Dementsprechend arbeitete er wie bereits bei den mittelalterlichen Herrschporträts mit Typologien, untersuchte diese epochen- und gattungsübergreifend sowie in europäischer Perspektive. Zudem dachte er bereits früh daran, sich die Kenntnisse von Experten zunutze zu machen, die ganze Kapitel zu Spezialfragen beisteuern sollten, verstand mitunter das Unternehmen im warburgschen Sinn als Problemsammlung. ${ }^{39}$

In einem Brief, den Schramm im Juli 1934 an den ein halbes Jahr zuvor nach London emigrierten Saxl sandte, berichtete er von der übergeordneten Absicht, die er mit diesem Projekt verfolgte. Schramm wollte "an der Geschichte der Herrschaftssymbole in den einzelnen Ländern und in den einzelnen Jahrhunderten die Geschichte des symbolischen Denkens" untersuchen. Saxl antwortete umgehend: "Ich finde Ihre Ausführungen über die HerrschaftsSymbole - wie Sie sich denken können - sehr interessant." ${ }^{40}$ Die Symbolimmung, auf die Schramms Vorhaben bei Saxl stieß, erstaunt Zustim nicht, kann dicse aus der Bibliotingen weilenden Warburg einst über Schramm und dem in Kreuzlingen weilender 1922 berichtet. Doch in das Gefühl dessen Vortrag vom Dezember 1922 berichtet. Doch in bereits der wissenschaftlichen Verbundenheit hate sich war "nälich ein damals Skepsis gemischt, denn Sch sind und ich bin", wie er Mensch ganz anderer Geistesart als Sie es sind und ichms Vorhaben im gleichen Brief an Warburg schrieb. An Schaftssymbole" lässt einer "Geschichte der mittelalterlichen Herron durch Warburg und zugleich die Entfremdung von den Mitarbeitern der nach London emigrierten Bibliothek besonders deutlich zeigen.

1938 erschien Berent Schwineköpers Studie Der Handschuh im Recht, Ämterwesen, Brauch und Volksglauben, als deren Göttinger Doktorvater Schramm eine Einleitung verfasst hatte. ${ }^{42}$ Hier verab-

39 Bei Erscheinen 1954-1956 schließlich hatten Wilhelm Berges, Albert Boeckler,

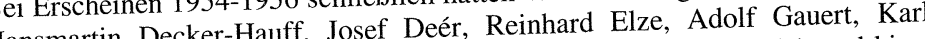
Hansmartin Hu unterschiedlich lange Kapitel verfasst.

40 Wu hierzu: Thimme, Percy Ernst Schramm, S. 443

41 WIA GC 1923, Mappe "Saxl/Warburg", F. Saxl an A. Warburg, Hamburg, 4. Jan. 1923.

42 Schwineköper, Der Handschuh im Recht. 
schiedete er sich radikal von dem oben entworfenen Forschungsvorhaben. Er verortete die Beschäftigung mit Herrschaftssymbolen nun ganz anders, als er es Saxl gegenüber wenige Jahre zuvor noch getan hatte. Nun knüpfte Schramm seinen Begriff mittelalterlicher Herrschaftssymbole nicht mehr an die umfassende philosophische Tradition des Warburg-Kreises, wie sie Ernst Cassirer für das Programm der Bibliothek im ersten Band der Vorträge 1921/1922 paradigmatisch formuliert hatte, ${ }^{43}$ sondern an die Forschung des Rechtshistorikers Karl von Amira und damit indirekt an Jacob Grimms Deutsche Rechtsaltertümer. Diese Tradition wollte Schramm nun für die Erforschung der politischen, rechtlichen und verfassungsgeschichtlichen Bedeutung der Herrschaftssymbole fruchtbar machen und verwarf hierfür sein bisheriges Verständnis des Symbolbegriffs. "Mit diesem Wort ist nicht weiter zu kommen, bevor ihm nicht eine feste, vom Gebrauch der Philosophie abgelöste Bedeutung zurückgewonnen ist. Bis dahin tut man besser es zu umgehen." ${ }^{44}$ Schramm sprach künftig anstelle von Herrschaftssymbolen von Herrschaftszeichen.

Seine Abkehr ist zwar unverkennbar gegen die begriffliche Tradition der Bibliothek gerichtet, doch stellte sie keinen plötzlichen Gesinnungswandel dar. Ebenso wenig hatte sie ihren Ursprung in dem im Januar 1935 von Schramm herbeigeführten Bruch mit den Mitarbeitern und Freunden, die weiterhin mit der Bibliothek Warburg verbunden blieben, allen voran Fritz Saxl, dann aber auch Edgar Wind und Raymond Klibansky. Anlass hierzu war bekanntlich die Einleitung Edgar Winds zur Kulturwissenschaftlichen Bibliographie zum Nachleben der Antike, die Wind - bereits aus der Emigration geschrieben, aber 1934 noch in Leipzig bei Teubner erschienen - zu einer Verknüpfung von politischen Entwicklungen in Deutschland und wissenschaftlicher Methode nutzte, in deren Nähe Schramm sich nicht gerückt sehen wollte. ${ }^{45}$

Es war nicht die Einschätzung des politischen Wandels von 1933 oder Schramms persönliches Verhältnis zum NS-Regime, sondern die methodische Konsequenz von Schramms wissen-

43 Cassirer, Der Begriff der symbolischen Form.

44 Einführung zu Schwineköper, Der Handschuh im Recht, S. XIII. Wieder abgedruckt in: Schramm, Kaiser, Könige und Päpste, Bd. 4.2, S. $667 \mathrm{f}$.

45 Der von Schramm herbeigeführte Bruch mit der Forschergemeinschaft der Bibliothek Warburg und mit dem ihm besonders freundschaftlich verbundenen Fritz Saxl soll hier nicht erneut dargestellt werden. Vgl. Grolle, Percy Ernst Schramm; Thimme, Percy Ernst Schramm, S. 373 f., S. 444 f. schaftlicher Sozialisation in der historischen Mediävistik seiner Zeit, die den Ausschlag gab, dass er sich von den Herrschaftssymbolen ab- und den Herrschaftszeichen zugewendet hatte. Im Bruch zwischen Schramm und den Bibliotheksmitarbeitern zeigt sich dieses wissenschaftshistorische Argument wie unter einem Prisma als Scheitern persönlicher Beziehungen und Freundschaften, als politische Positionsbezüge und als biografische Schicksale. Damit soll weder die Dramatik noch die existenzielle Bedrohung, die für die Bibliothek und deren Angehörigen mit dem Jahr 1933 verbunden waren, geschmälert oder gar in Abrede gestellt werden. Vielmehr geht es darum, die Frage nach der Entwicklung des Faches jenseits individueller Verhaltensweisen als Ausdruck wissenschaftsgeschichtlicher Bedingungen und Positionen zu verstehen und somit von der Ebene einer Personengeschichte auf diejenige von Wissenschaftskultur zu wechseln.

Denn bereits im Sommer 1934 und somit ein gutes halbes Jahr vor dem Konflikt, als er Saxl über sein Vorhaben "einer Geschichte der Herrschaftssymbole als einer Geschichte des symbolischen Denkens" berichtete, schrieb er relativierend, es dränge ihn "doch immer wieder zur politischen und Verfassungsgeschichte zurück." ${ }^{46}$ In diesem Brief zeigt sich die Spannung einer doppelten, letztlich nicht aufzulösenden wissenschaftshistorischen Sozialisation Schramms besonders deutlich; auf den Pfaden warburgscher Forschung nach Bildern als kulturelle Schemata einerseits, in der Tradition deutscher Reichsmediävistik, deren Interesse am 'Wesen des Staates' und der Suche nach dem Spezifischen der deutschen Kultur andererseits. Diesen beiden Prägungen lag ein unterschiedliches Bildverständnis zugrunde, unterschiedliche Arten, sich von Bildern affizieren zu lassen, die ihrerseits Gegenstandswahl, Hypothesenbildung und Argumentationsanlage bestimmten.

In Herrschaftszeichen und Staatssymbolik zeigt sich Schramms gewandeltes Bildverständnis sehr deutlich. Die Herrschaftszeichen werden nicht mehr als Schemata kultureller Erinnerung und ihrer Tradierung verstanden, sondern als der tangible Beweis dafür, dass bereits im frühen Mittelalter Herrschaftsvorstellungen existierten, die man als 'staatliche' Gebilde bezeichnen kann. Der Bedeutungsgehalt des Bildes steht somit nicht mehr zur Disposition, unterliegt auch kaum einer Veränderung, sondern ist genau, präzise und ex-

46 WIA, GC 1934-1936, P. E. Schramm an F. Saxl, Göttingen, 9. Juli 1934. 
klusiv auf die Herrschaft und den 'Staat' gerichtet und gedeutet. Indem er Bild- und Textquellen konsequent aufeinander bezog, überbrückte Schramm manche Überlieferungslücke geschickt. Damit gelingt es ihm, mittelalterliche Herrschaftsvorstellungen als rückprojizierte Kontinuitäten bis ins dritte nachchristliche Jahrhundert nachzuweisen und somit den 'mittelalterlichen Staat' tief in der Geschichte zu verankern. "Wenn wir uns mit der Geschichte der Herrschaftszeichen und der Staatssymbolik befassen," so Schramm im Schlusskapitel, "vermögen wir also bereits die ersten Phasen des 'Staates' zu erhellen und die Kenntnis, die wir auf Grund der Annalen, Urkunden und Briefe von den folgenden besitzen zu vertiefen." ${ }^{47}$ Es ging Schramm also darum, in der Analyse der Zeichen hinter die schriftlichen Zeugnisse, in denen für das Früh- und Hochmittelalter kein Begriff 'Staat' überliefert ist, zum 'Wesen' und somit zur eigentlichen Geschichte zu gelangen, die sich auf dieser Ebene bis zur Frage nach dem 'deutschen Sonderweg' fortschrieb.

Schramms Auseinandersetzung mit seinen beiden Sozialisationen ist unverkennbar, deutlich aber auch die Seite, der er schließlich zuneigte. Seine Verwerfung des philosophischen Symbolbegriffs nach Cassirer war ja nur möglich, weil er damit aufs Beste vertraut war, weil er selbst seine Geschichte mittelalterlicher Herrschaft zunächst in dieser Perspektive unternehmen wollte. Sich vom Symbol abzuwenden und an dessen Stelle den Begriffs des 'Zeichens' oder 'Sinnzeichens' zu setzen, führte die Analyse mittelalterlicher Herrschaftspräsentation an sich noch nicht weiter. Die Begriffe waren nicht trennschärfer oder präziser, doch sie waren nicht mit den methodischen Konnotationen kulturwissenschaftlicher Bildforschung im Geiste Warburgs und dessen Bibliothek befrachtet, die für Schramms Interessen an der politischen und Verfassungsgeschichte des Mittelalters nicht weiterführend waren. Anstatt die mittelalterlichen Herrschaftssymbole zu einer Geschichte des symbolischen Denkens zusammenzufassen, untersuchte Schramm nun die Herrschaftszeichen auf ihre Bedeutung für einen mittelalterlichen Staat und die Herrschaft in einem 'deutschen Reich' hin. Der Wandel vom Symbol zum Zeichen steht somit für einen Entscheid gegen die methodische Inspiration durch die Kulturtheorie Aby Warburgs und für eine endgültige Hinwen-

\footnotetext{
47 Schramm, Herrschaftszeichen und Staatssymbolik, S. 1068.
}

dung zur Tradition deutscher Reichsmediävistik und deren Fortführung im Bereich einer Historiografie, die sich bildlicher Quellen bediente.

Für die Entwicklung einer historischen Bildforschung war Schramms Verengung des Bildverständnisses hin auf die Bedeutung von Bildern als eindeutige Zeichen von Herrschaft hinderlich. Die Frage nach Bildzeugnissen wurde nicht mehr als Frage nach Kultur, ihrer Tradierung und ihrem Wandel, sondern als Frage von 'Staat', seiner Begründung und Kontinuität gestellt. Gegenüber Warburgs Theorie eines kulturellen Bildgedächtnisses bedeutete dies eine markante Beschränkung, die die Adaption bildlicher Quellen auf andere Forschungsanliegen zumindest nicht beförderte.

\section{Verklärte Erinnerung und die Zukunft des Faches}

In Schramms Übergang vom Symbol zum Zeichen spiegelt sich jedoch nicht nur ein individueller Entscheid, sondern auch die Entwicklung des Faches, die Wirkkraft historiografischer Traditionen und schließlich die Konsequenzen für eine Bildforschung innerhalb der Geschichtswissenschaft. Anne Nagel hat jüngst gezeigt, wie viel Vergangenheit in der Zukunft eines Faches liegt und wie sehr dies auch auf die historische Mediävistik zutrifft. Die Kontinuitäten der Themen, Methoden, Institutionen und schließlich Akteure mittelalterlicher Historiografie, die das Fach losgelöst von einer direkten Einflussnahme der gesellschaftlichen und politischen Umwälzungen und Veränderungen im Jahrhundert zwischen 1860 und 1960 prägten und immer noch prägen, sind gleichermaBen eindrücklich wie erstaunlich. Für die Geschichtswissenschaft im allgemeinen, die historische Mediävistik im speziellen gilt, dass die methodischen Anregungen der Kulturtheorie Aby Warburgs kaum rezipiert wurden und, da mit den Kontinuitäten des Faches nicht konstruktiv zu verbinden, die Entstehung einer historischen Bildforschung bis in die jüngste Zeit nicht zu prägen vermochten.

Selbstverständlich beschäftigte sich Percy Ernst Schramm, der unter den Mittelalterhistorikern als einer der ersten und möglicherweise am intensivsten Warburgs Überlegungen an die Geschichte des Mittelalters anzulegen versuchte, weiterhin mit Bildern. Einiges an seinem Umgang mit Bildern erinnert bis heute an 
die Prägung durch Warburg. Sein Erkenntnisinteresse hat sich jedoch markant von Warburgs Forschungsvorhaben wegentwickelt; sein Bildverständnis schließlich muss als von Warburg grundsätzlich verschieden bezeichnet werden. Denn von den Dimensionen symbolischer und psychologischer Bildbedeutung, von einer Vorstellung von Bildern als Medien kultureller Energieströme und einer Bildwissenschaft als deren analytischen Durchdringung, bei der dennoch stets ein letzter Rest von Bildmagie übrig bleibt, also von all denjenigen Facetten, die Warburg zum Begründer einer Theorie des kulturellen Bildgedächtnisses machen, das im ausgehenden 20. Jahrhundert von zahlreichen Disziplinen neu entdeckt und aufgegriffen wurde, hatte sich Percy Ernst Schramm entfernt, bevor er sich davon lossagte.

Für die Entwicklung einer historischen Bildforschung wirkte Percy Ernst Schramm letztlich befördernd und hemmend zugleich. Einerseits etablierte er die Berücksichtigung bildlicher Quellen, insbesondere der Herrschaftszeichen und Herrscherporträts jenseits hilfswissenschaftlicher Traditionen als festen Bestandteil der historischen Mediävistik; andererseits trugen seine Arbeiten durch ihr Erkenntnisinteresse, ihr Bildverständnis und ihre Methode zu einer Verengung der Analyse von Bildquellen auf deren staatliche Bedeutung bei und trugen somit zur Verzögerung einer inhaltlichen und methodischen Rezeption von Warburgs Anregungen in der Geschichtswissenschaft bei. Die von Schramm selbst ja zeitlebens nicht nur eingestandene, sondern emphatisch betonte Inspiration durch Aby Warburg mag die Rezeption des Hamburger Privatgelehrten in der deutschsprachigen Geschichtswissenschaft für andere Perspektiven zusätzlich erschwert haben, denn Warburg war durch Schramm gleichsam für dessen eigene Sicht auf das Verhältnis von Bild und Geschichte besetzt. ${ }^{48}$

In seiner persönlichen Erinnerung führte Percy Ernst Schramm sein Interesse an "Bildern als Geschichtsdokumenten" auf die Begegnung mit Aby Warburg zurück; das ist sicherlich richtig. Damit zugleich Warburgs Grundproblem des 'Nachlebens der Antike' und

48 Es ist deswegen bezeichnend, dass die fächerübergreifende Relevanz des warburgschen Bildgedächtnisses und dessen Bedeutung für die Geschichtswissenschaft außerhalb der deutschsprachigen Geschichtswissenschaft bereits viel früher erkannt worden war. Bereits 1966 hatte Carlo Ginzburg hierzu einen Beitrag verfasst. Vgl. Ginzburg, Da Warburg a E. H. Gombrich. dessen methodisches Verständnis zum Ausgangspunkt und zur Inspiration für die eigene Forschung - wenn auch nur implizit - zu erklären, ist mit gleicher Sicherheit verfehlt, denn Schramm entwickelte sich und seine Forschungsinteressen in ganz andere Richtungen. Doch von dieser Überzeugung rückte Schramm bis zu seinem Tod 1970 nicht ab. Noch 1968 schrieb er: "Dankbar bekenne ich mich am Schluss dieser Ausführungen zu den Anregungen, die ich in bezug auf die Aussagekraft von Bildern von meinem Lehrer ABY WARBURG (1866-1929) empfing." ${ }^{49}$ Es ist signifikanter Teil dieser verklärenden Sicht auf die eigene wissenschaftliche Biografie, dass Schramm, als er sich gegen Ende seines Lebens an den Vortrag erinnerte, den er 1922 in der Bibliothek Warburg hielt, der Überzeugung war, dass damals auch "sein Lehrer Aby Warburg" zugehört hätte..$^{50}$ Sowohl aus der im Warburg Institute verwahrten Korrespondenz als auch aus der jüngst herausgegebenen Kreuzlinger Krankenakte Warburgs wird ersichtlich, dass das mit Sicherheit nicht der Fall gewesen ist. ${ }^{51}$ Schramms Bemühen, die Prägung durch Warburg derart zu betonen, ist nicht nur verklärend, sondern diente ihm auch dazu, sich die Brüche seiner eigenen Biografie als Kontinuitäten zu erklären. An dieser Stelle fallen die Entwicklungen des Faches, seiner Institutionen und der Forscher erneut ineinander und müssen als Problemgeschichte doch unterschieden werden. Für Percy Ernst Schramm und die Frage nach dessen Beitrag zu einer historischen Bildforschung als moderner Kulturwissenschaft stellt sie sich heute als doppelte Verwerfung dar. Als Verwerfung methodischer Inspiration durch Aby Warburgs Theorie eines kulturellen Bildgedächtnisses und als Verwerfung persönlicher Beziehungen zum Kreis der Mitarbeiter an der Bibliothek Warburg im Jahre 1935.

\footnotetext{
49 Schramm, Kaiser, Könige und Päpste, Bd. I, S. 29.

50 Thimme, Percy Ernst Schramm, S. 137.

51 Marazia/Stimilli, Ludwig Binswanger.
} 


\section{Literatur}

\section{Ungedruckte Quellen}

WIA [Warburg Institute Archive], GC [General Correspondance] 1922, P. E. Schramm an F. Saxl, Heidelberg, 3. April 1922

- GC 1922, P. E. Schramm an A. Warburg, Hamburg, 5. Okt. 1922

- GC 1923, Mappe "Saxl/Warburg", F. Saxl an A. Warburg, Hamburg, 4. Jan. 1923

- GC 1921, F. Saxl an W. Printz, Hamburg, 12. Dez. 1921

- GC 1934-1936, P. E. Schramm an F. Saxl, Göttingen, 9. Juli 1934

\section{Literatur}

Burkart, Lukas, "Die Träumereien einiger kunstliebender Klosterbrüder ...". Zur Situation der Kulturwissenschaftlichen Bibliothek Warburg zwischen 1929 und 1933, in: Zeitschrift für Kunstgeschichte, 63, 2000, H. 1, S. 89-119

Cassirer, Ernst, Der Begriff der symbolischen Form im Aufbau der Geisteswissenschaften, in: Saxl, Fritz (Hg.), Vorträge der Bibliothek Warburg, Bd. I, Vorträge 1921-1922, Leipzig 1923, S. 11-39

Geuenich, Dieter/Oexle, Otto Gerhard (Hg.), Memoria in der Gesellschaft des Mittelalters, Göttingen 1994

Ginzburg, Carlo, Da Warburg a E. H. Gombrich (Note su un problema del metodo), in: Studi medievali, 7, 1966, S. 1015-1065 [Deutsch: Kunst und soziales Gedächtnis. Die Warburg-Tradition, in: Ders., Spurensicherungen. Über verborgene Geschichte, Kunst und soziales Gedächtnis, München 1983, S. 149-233]

Goethe, Johann Wolfgang v., Nachträgliches zu Philostrats Gemählden, in: Weimarer Ausgabe, 1. Abtheilung, 49. Band, 1. Abtheilung, Weimar 1898

Gombrich, Ernst H., Aby Warburg. An intellectual Biography, London 1970

Groebner, Valentin, Das Mittelalter hört nicht auf. Über historisches Erzählen, München 2008

Grolle, Joist, Percy Ernst Schramm - Fritz Saxl. Die Geschichte einer zerbrochenen Freundschaft, in: Zeitschrift des Hamburger Geschichtsvereins, 76, 1990, S. 145-167

Hampe, Karl, Deutsche Kaisergeschichte in der Zeit der Salier und Staufer, Heidelberg 1949

Haskell, Francis, Die Geschichte und ihre Bilder. Die Kunst und die Deutung der Vergangenheit, München 1995

Kaemmerling, Ekkehard, Ikonographie und Ikonologie. Theorien - Entwicklung - Probleme. Bildende Kunst als Zeichensystem, Köln 1987
Le Goff, Jacques, Geschichte und Gedächtnis [frz. 1988], Frankfurt/M. 1992

Marazia, Chantal/Stimilli, Davide (Hg.), Ludwig Binswanger - Die unendliche Heilung. Aby Warburgs Krankengeschichte, Zürich 2007

Nagel, Anne Christine, Im Schatten des Dritten Reichs. Mittelalterforschung in der Bundesrepublik Deutschland 1945-1970, Göttingen 2005

Oexle, Otto Gerhard (Hg.), Memoria als Kultur, Göttingen 1995

- Geschichtswissenschaft im Zeichen des Historismus. Studien zur Problemgeschichte der Moderne, Göttingen 1996

- Der Blick auf die Bilder. Kunstgeschichte und Geschichte im Gespräch, Göttingen 1997

- (Hg.), Das Problem der Problemgeschichte 1880-1932, Göttingen 2001

- "Staat" - "Kultur" - "Volk". Deutsche Mittelalterhistoriker auf der Suche nach der historischen Wirklichkeit 1918-1945, in: Moraw, Peter/Schieffer, Rudolf (Hg.), Die deutschsprachige Mediävistik im 20. Jahrhundert, Ostfildern 2005, S. 63-101

Raulff, Ulrich, Parallel gelesen: die Schriften von Aby Warburg und Marc Bloch zwischen 1914 und 1924, in: Bredekamp, Horst u. a. (Hg.), Aby Warburg. Akten des internationalen Symposions Hamburg 1990, Weinheim 1991, S. 167-178

Roeck, Bernd, Psychohistorie im Zeichen Saturns. Aby Warburgs Denksystem und die moderne Kulturgeschichte, in: Hardtwig, Wolfgang/Wehler, Hans-Ulrich (Hg.), Kulturgeschichte heute, Göttingen 1996, S. 231-254

Saxl, Fritz, Die Kulturwissenschaftliche Bibliothek Warburg in Hamburg [1930], in: Aby Warburg, Ausgewählte Schriften und Würdigungen, hrsg. von Dieter Wuttke, Baden-Baden 1979, S. 331-334

Schieffer, R., Weltgeltung und nationale Verführung. Die deutschsprachige Mediävistik vom ausgehenden 19. Jahrhundert bis 1918, in: Moraw, Peter/Schieffer, R. (Hg.), Die deutschsprachige Mediävistik im 20. Jahrhundert, Ostfildern 2005, S. 39-61

Schramm, Percy Ernst, Das Herrscherbild in der Kunst des frühen Mittelalters, in: Saxl, Fritz (Hg.), Vorträge der Bibliothek Warburg, Bd. 2, Vorträge 1922-1923, Teil I, Leipzig 1924, S. 145-224

- Herrschaftszeichen und Staatssymbolik. Beiträge zu ihrer Geschichte vom dritten bis zum sechzehnten Jahrhundert, Bd. 1, Stuttgart 1954

- Kaiser, Könige und Päpste. Beiträge zur allgemeinen Geschichte, 1. Bd., Stuttgart 1968

- Kaiser, Könige und Päpste. Gesammelte Aufsätze zur Geschichte des Mittelalters, 4 Bde., Stuttgart 1968-1971

- Neun Generationen. Dreihundert Jahre deutscher "Kulturgeschichte" im Lichte der Schicksale einer Hamburger Bürgerfamilie (1648-1948), 2. Bd., Göttingen 1964

- Sphaira - Globus - Reichsapfel. Wanderung und Wandlung eines Herrschaftszeichens von Caesar bis Elisabeth II. Ein Beitrag zum "Nachle- 
ben der Antike", Stuttgart 1958 (wieder abgedruckt in: Schramm, Kaiser, Könige und Päpste, Bd. 4,2)

Schwineköper, Berent, Der Handschuh im Recht, Ämterwesen, Brauch und Volksglauben, Berlin 1938

Thimme, David, Percy Ernst Schramm und das Mittelalter. Wandlungen eines Geschichtsbildes, Göttingen 2006

Tolkemitt, Brigitte/Wohlfeil, Rainer (Hg.), Historische Bildkunde. Probleme - Wege - Beispiele (Zeitschrift für Historische Forschung, Beiheft 12), Berlin 1991

Warburg, Aby, Gesammelte Schriften. Studienausgabe, hrsg. von Horst Bredekamp u. a., 1. Abt., Bd. I,1, Berlin 1998

- Italienische Kunst und internationale Astrologie im Palazzo Schifanoja zu Ferrara, in: Ders., Gesammelte Schriften, hrsg. von Horst Bredekamp u. a., 1. Abt., Bd. I,2, Berlin 1998, S. $478 \mathrm{f}$.

- Tagebuch der Kulturwissenschaftlichen Bibliothek Warburg mit Einträgen von Gertrud Bind und Fritz Saxl [Michels, Karen/Schoell-Glass, Charlotte (Hg.), Aby Warburg, Gesammelte Schriften. Studienausgabe, 7. Abt., Bd. 7], Berlin 2001

\section{MARTIN KNAUER}

\section{Drei Einzelgänge(r): Bildbegriff und Bildpraxis der deutschen Historiker Percy Ernst Schramm, Hartmut Boockmann und Rainer Wohlfeil (1945-1990)}

Am 14. Oktober 1954 beging Percy Ernst Schramm seinen 60. Geburtstag. Zu diesem Anlass überreichten ihm seine Göttinger Schüler, initiiert von Gudrun Colsmann und Norbert Kamp, eine handgeschriebene, mit Fotografien ausgestattete Festschrift. ${ }^{1}$ Grundlage des kleinen Albums bildet eine ironische Auseinandersetzung mit Schramms Bildern der Woche, einem seiner "Lieblingskinder", wie es in der Widmung an den Jubilar heißt. Beim Konzept des im Singular gebrauchten - Bildes der Woche handelt es sich um eine Zusammenstellung von bis zu acht Abbildungen, die gemeinsam mit dem ein- bis zweiseitigen Kommentar auf einem Schwarzen Brett montiert wurden. Das Spektrum dieser Bilderschauen, deren Motive aus Katalogen und Zeitschriften stammten und deren Anbringung Schramms Mitarbeiter organisierten, ${ }^{2}$ lässt sich anhand der sechs Bildwände gut rekonstruieren: "Die d(t)ämonische Frau im Jugendstil", "Menschen der Reformationszeit. Skizzen Holbeins d. J.", "Köpfe (Italiener des 15. Jahrhunderts)", "Der Teppich von Bayeux" und "Funde aus Trier". Das letzte im Album aufgenommene Beispiel ist betitelt: "...ohne Bilder". Es zeigt und hier kommt die Ironie ins Spiel - lediglich eine große Lateinamerika-Karte aus der Weltschau vom 10. April 1954. ${ }^{3}$

StA Hamburg, Best. 622-1 Schramm. Hier: Nachlieferung der Universität Göttingen; Karton 1, grauer Halbordner, Aufschrift "Thron". Prof. Joist Grolle hat mich freundlicherweise auf diesen noch uninventarisierten Bestand aufmerksam gemacht. Ich danke Prof. Gottfried Schramm und Volker Reißmann (Staatsarchiv Hamburg) für die Erlaubnis zur Einsichtnahme.

2 Auskunft Prof. Joist Grolle, Mai 2006.

3 Vollständiger Titel: "Weltschau: Südamerika - ein Kontinent in Wandlung, 10. April 1954". 
Jens Jäger · Martin Knauer (Hrsg.)

\section{Bilder als historische Quellen?}

Dimension der Debatten um historische Bildforschung 
Bibliografische Information der Deutschen Nationalbibliothek

Die Deutsche Nationalbibliothek verzeichnet diese Publikation in der Deutschen Nationalbibliografie; detaillierte bibliografische Daten sind im Internet über http://dnb.d-nb.de abrufbar.

Gedruckt auf umweltfreundlichem, chlorfrei gebleichtem und alterungsbeständigem Papier.

Alle Rechte, auch die des auszugsweisen Nachdrucks, der fotomechanischen Wiedergabe und der Übersetzung, vorbehalten. Dies betrifft auch die Vervielfältigung und Übertragung einzelner Textabschnitte, Zeichnungen oder Bilder durch alle Verfahren wie Speicherung und Übertragung auf Papier, Transparente, Filme, Bänder, Platten und andere Medien, soweit es nicht $\S 53$ und 54 URG ausdrücklich gestatten.

() 2009 Wilhelm Fink Verlag, München

(Wilhelm Fink GmbH \& Co. Verlags-KG, Jühenplatz 1, D-33098 Paderborn)

Internet: www.fink.de

Einbandgestaltung: Evelyn Ziegler, München Printed in Germany.

Herstellung: Ferdinand Schöningh GmbH \& Co. KG, Paderborn
JENS JÄGER/MARTIN KNAUER

Bilder als historische Quellen? Ein Problemaufriss

DANA ARNOLD

Sehen heißt glauben: Historiker und Bilder.

\section{JENS JÄGER}

Zwischen Bildkunde und Historischer Bildforschung

- Historiker und visuelle Quellen 1880-1930.

\section{LUCAS BURKART}

Verworfene Inspiration. Die Bildgeschichte Percy Ernst

Schramms und die Kulturwissenschaft Aby Warburgs

\section{MARTIN KNAUER}

Drei Einzelgänge(r): Bildbegriff und Bildpraxis der deutschen Historiker Percy Ernst Schramm, Hartmut Boockmann und Rainer Wohlfeil (1945-1990)

\section{GERHARD PAUL}

Die aktuelle Historische Bildforschung in Deutschland.

Themen - Methoden - Probleme - Perspektiven

DANIELA KNEISSL

L'historien saisi par l'image: Bildzeugnisse als

Forschungsgegenstand in der französischsprachigen

Geschichtswissenschaft des 20. Jahrhunderts

Autorenverzeichnis

Personenregister 